\title{
A Review on Metabolism and Cancer in Relation with Circadian Clock Connection
}

\author{
Merlin Jayalal .L .P
}

\begin{abstract}
Circadian rhythms govern a remarkable variety of metabolic and physiological functions. Accumulating epidemiological and genetic evidence indicates that the disruption of circadian rhythms might be directly linked to cancer. Intriguingly, several molecular gears constituting the clock machinery have been found to establish functional interplays with regulators of the cell cycle, and alterations in clock function could lead to aberrant cellular proliferation. In addition, connections between the circadian clock and cellular metabolism have been identified that are regulated by chromatin remodelling. This suggests that abnormal metabolism in cancer could also be a consequence of a disrupted circadian clock. Therefore, a comprehensive understanding of the molecular links that connect the circadian clock to the cell cycle and metabolism could provide therapeutic benefit against certain human neoplasias.
\end{abstract}

Key words : Circadian Rhythm, Cancer, CLOCK, BMAL1, Cryptochrome

\section{Introduction}

The process of tumorigenesis is intimately linked to the disruption of the balance that characterizes homeostasis $^{[1]}$. Independent of the causal agents, it is now accepted that the aberration of specialized metabolic pathways may contribute to cell transformation. Cancer cells predominantly use glycolysis rather than mitochondrial oxidative phosphorylation for energy production, which is known as the Warburg effect ${ }^{[2]}$. Although the molecular gears that intersect metabolic control and the aberrant cellular proliferation that is characteristic of tumour cells are not fully deciphered ${ }^{[3]}$, several intriguing hints indicate that the cellular machinery that governs circadian physiology is likely to occupy a strategic signalling position ${ }^{[4,5]}$. This idea is particularly relevant as emerging evidence has revealed tight links between the molecular clock and the regulation of cellular metabolism ${ }^{[6]}$.

The Earth's rotation around its axis leads to daynight cycles, which affects the physiology of most living organisms. Circadian (from the Latin circa diem

Department of Biochemistry, Bharathidasan college of Arts and Science, Ellispettai, Erode-638116, Tamilnadu, India

${ }^{\dagger}$ Corresponding author : 1pmjlal@gmail.com

(Received : August 13, 2012 Revised : September 20, 2012, Accepted : September 25, 2012) meaning 'about a day') clocks are intrinsic, time-tracking systems that enable organisms to anticipate environmental changes (such as food availability and predatory pressure) and allow them to adapt their behaviour and physiology to the appropriate time of day ${ }^{[7]}$. Rhythms that occur with a periodicity of 24 hours are considered circadian. Feeding behaviour, sleep-wake cycles, hormone levels and body temperature are just a few examples of physiological circadian rhythms, with light being the principal regulator or zeitgeber. The three integral parts of circadian clocks are an input pathway that includes detectors to receive environmental cues (or zeitgebers) and transmit them to the central oscillator, a central oscillator that keeps circadian time and generates rhythm, and output pathways through which the rhythms are manifested by the control of various metabolic, physiological and behavioural processes. The distinguishing characteristics of circadian clocks are that they are entrainable (that is, circadian clocks can be synchronized by external cues), self-sustained (oscillations can persist even in the absence of environmental cues) and temperature compensated (meaning that moderate variations in ambient temperature do not affect the period of circadian oscillation $)^{[8]}$.

A major consequence of a modern lifestyle is the disruption of circadian rhythms. This leads to several pathological conditions, including sleep disturbances and 
depression. Importantly, accumulating evidence shows that the alteration of circadian rhythms might lead to increased susceptibility to cancer in humans. Epidemiological studies have revealed that the risk for breast cancer is significantly higher in industrialized societies, and that the risk increases as developing countries become more westernized ${ }^{[9]}$. Moreover, a moderate increase in the incidence of breast cancer is reported in women who work night shifts, and the incidence is higher among individuals who spend more hours per week and years working at night ${ }^{[10,11]}$. Exposure to light at night and the subsequent reduction in melatonin levels has been postulated to be a causal factor for this effect $^{[9]}$. Importantly, although the correlation between lower melatonin levels and higher incidence of breast cancer was observed in one study ${ }^{[12]}$, another group failed to observe such a correlation ${ }^{[13]}$. However, from a clinical point of view, cancer prognosis is poorer in patients with an altered circadian rhythm than in patients with a normal rhythm ${ }^{[14]}$. In addition, chronotherapy (drug administration based on the time of the day) has been shown to improve the efficacy of some chemotherapeutic agents ${ }^{[15,16]}$, underscoring the importance of understanding circadian rhythmicity. In this Review, we discuss the molecular mechanisms whereby the circadian clock regulates the cell cycle, DNA damage responses, ageing and metabolism. Aberrant circadian rhythms could lead to defects in the regulation of these processes, which might result in tumorigenesis and tumour progression.

\section{Regulation of the Circadian Clock in Mammals}

Circadian clocks are present in almost all mammalian tissues. The master or central clock is located in the hypothalamic suprachiasmatic nucleus ( $\mathrm{SCN}$ ), a small brain region containing 10,000-15,000 neurons. The SCN clock can function autonomously, without any external input, but it can be reset by environmental cues such as light. Clocks outside the SCN are referred to as peripheral clocks and are thought to be synchronized by the master clock to ensure temporally coordinated physiology ${ }^{[6]}$. The synchronization mechanisms implicate various humoral signals, including circulating entraining factors such as glucocorticoids ${ }^{[17]}$. Moreover, peripheral clocks, such as the liver, can be synchronized by the availability of metabolites or feeding time ${ }^{[18]}$. Peripheral clocks are present in almost all mammalian tissues, such as the liver, heart, lungs and kidneys, where they maintain circadian rhythms and modulate transcription factors in a paracrine fashion to regulate tissue-specific gene expression. For example, the liver clock controls the expression of several metabolic genes, Such as phosphoenolpyruvate carboxykinase 2 (PCK2; which encodes a gluconeogenic enzyme), the activity of which is decreased in hepatic cancer ${ }^{[19]}$.

\subsection{The Negative Feedback Loop.}

From a molecular standpoint, circadian rhythms are regulated by transcriptional and post-translational feedback loops generated by a set of interplaying clock proteins. The fundamental mechanism of the generation and maintenance of rhythms is similar in the central and the peripheral clocks; however, the output pathways elicited can be different and more tissue specific. The positive limb of the mammalian clock machinery is comprised of CLOCK and bMAL1, which are transcription factors that hetero dimerize through the PAS domain and induce the expression of clock-controlled genes by binding to their promoters at E-boxes. The Cryptochrome (Cryl and Cry2) and period (Per1, Per2 and Per3) families are clock-controlled genes and encode proteins that negatively regulate the circadian machinery. Per and Cry proteins are thought to translocate into the nucleus and form a complex to inhibit CLoCK-bMAL1-mediated transcription, thereby closing the negative feedback loop6. To start a new transcription cycle, the CLoCK-bMAL1 complex needs to be de-repressed through the proteolytic degradation of Per and Cry. Although we have described the mammalian circadian clocks, the generation and maintenance of rhythms is conceptually conserved in other model organisms such as Drosophila melanogaster, Neurospora crassa and plants ${ }^{[20]}$.

The CLoCK-bMAL1 complex activates the clock output by inducing the expression of a large number of genes. Genomic studies have shown that the molecular output of the circadian clock is remarkable as up to $10 \%$ of all cellular transcripts oscillate in a circadian manner ${ }^{[21]}$ - a large proportion of which seem to be directly regulated by CLCK-bMAL1. Although it is important to emphasize that many clock-controlled genes encode proteins crucial in regulating cellular out- 
put and metabolic and physiological functions ${ }^{[22]}$, some of the output products can also feed back to the clock machinery ${ }^{[23,24]}$.

Resetting of the circadian clock through stimuli such as light (in the SCN) or serum shock (in fibroblasts) is associated with a rapid induction of Perl and Per2 $\mathrm{mRNA}^{[25-27]}$. Although it is not clearly understood how light or other entraining cues directly affect CLoCKbMAL1, the MAP kinases ${ }^{[28]}$ (which are known to phosphorylate bMAL1 ${ }^{[29]}$ and CLoCK phosphorylation by protein kinase $\mathrm{C}^{[30]}$ have been implicated in this process.

The core clock machinery is more complex than a single auto regulatory loop: several interconnected feedback loops operate in concert. Some are transcriptional and post-translational, others involve the proteasome and/or enzymatic metabolic loops ${ }^{[31,32]}$. For example, Bmall is regulated by the orphan nuclear receptors retinoic acidrelated orphan receptor- $\alpha(\operatorname{RoR} \alpha)$ and REV-ERbá, both of which are controlled by the circadian clock. Both of these proteins are transcription factors that bind to the Bmall promoter at REV-ERb $\alpha$ and RoRá response elements. RoRá activates Bmall transcription $^{[33]}$, whereas REV-ERbá inhibits it ${ }^{[34]}$ other core members of the mammalian circadian clock include neuronal PAS-domain protein 2 (NPAS2) and the enzymes casein kinase $\mathrm{I} \varepsilon(\mathrm{CKI} \varepsilon)$ and $\mathrm{CKI} \delta$. NPAS2 is a paralogue of CLoCK, exhibiting similar activities but differing in tissue distribution. CLoCK is ubiquitous but NPAS2 is mainly expressed in the forebrain ${ }^{[35]}$. NPAS2 can heterodimerize with bMAL1, bind to E-box motifs and transcriptionally activate circadian genes ${ }^{[35]}$. Recent reports suggest that NPAS2 can functionally compensate for $\mathrm{CLoCK}$ in the $\mathrm{SCN}^{[36]}$ but not in the periphery ${ }^{[37]}$, supporting the notion that CLoCK has a prominent role in metabolic tissues ${ }^{[38]}$.

\subsection{Post-translational Modifications}

Post-translational modifications (such as phosphorylation, acetylation, sumoylation and ubiquitylation) that are induced by various intracellular signalling pathways have a crucial role in the maintenance of circadian rhythms, for example Cry-mediated inhibition involves reduced phosphorylation of CLoCK and bMAL1 ${ }^{[39,40]}$. $\mathrm{CKI} \delta, \mathrm{CKI} \varepsilon$ and the $D$. melanogaster homologue doubletime $(\mathrm{dbT})$ have important roles in the regulation of circadian rhythms by phosphorylating Per proteins. Phosphorylation of Per proteins by CKI $\varepsilon$ and CKI $\delta$ leads to their ubiquitin-mediated degradation ${ }^{[41,42]}$. Conversely, Cry ubiquitylation, mediated by the SKP1-cullin-F box (SCF) - FbXL3 ubiquitin ligase, regulates Cry protein levels ${ }^{[43-45]}$. Sumoylation of bMAL1 regulates its stability ${ }^{[46]}$, whereas acetylation of bMAL1 enhances its interaction with $\mathrm{Cry}^{\left[{ }^{[4]}\right.}$. These modifications have rhythmicity, and mutations that abolish these modification sites impair the robustness of circadian rhythms ${ }^{[46,47]}$.

\subsection{Chromatin Remodelling}

Another level of circadian machinery regulation is achieved through chromatin remodelling. Circadian promoters have oscillations in histone modifications, such as acetylation ${ }^{[48]}$ and methylation ${ }^{[49]}$. Importantly, CLoCK also possesses Histone acetyltransferase (HAT) activity, which is essential for circadian clock-controlled gene expression ${ }^{[50]}$. As CLoCK binds to E-box regions of DNA, the HAT activity of CLoCK could selectively remodel chromatin at the promoters of clock-controlled genes. The enzymatic activity of CLoCK also allows it to acetylate non-histone substrates. For example, CLoCK mediates acetylation of its own binding partner, bMAL1, on Lys537 $7^{[47]}$. This acetylation is essential for circadian clock-controlled gene expression. Ectopic expression of wild-type bMAL1, but not an acetylationresistant bMAL1 mutant (K537R), is able to rescue the circadian expression of endogenous target genes in mouse embryonic fibroblasts (MEFs) derived from Bmal1-/- mice. The bMAL1-K537R mutant has drastically reduced sensitivity to CRY1-mediated repression compared with wild type bMAL1, indicating that the acetylation of bMAL1 by CLoCK might be an essential regulatory switch as it facilitates Cry-dependent repres$\operatorname{sion}^{[47]}$.

Recently, the histone deacetylase sirtuin 1 (SIRT1) was identified as a crucial modulator of the circadian clock machinery ${ }^{[51,52]}$. The activity of SIRT1 from mouse liver or synchronized fibroblasts has robust circadian rhythm. Moreover, SIRT1 regulates circadian rhythms by counteracting the HAT activity of CLoCK51. Histone H3 Lys9 and Lys14 at circadian promoters and bMAL1 are specific targets of SIRT1, which are hyperacetylated in Sirt1-/- MEFs. The CLoCK-bMAL1 complex interacts with SIRT1 and recruits it to the circadian promoters. Importantly, circadian gene expression and bMAL1 acetylation are compromised in liver-specific SIRT1- mutant mice 
(which lack exon 4, which encodes the catalytic domain of SIRT1 $)^{[51]}$. These findings led to the concept that SIRT1 operates as a rheostat of the circadian machinery, modulating the amplitude and 'tightness' of CLoCKmediated acetylation and consequent transcription cycles in metabolic tissues ${ }^{[51]}$. Moreover, the prominent role of SIRT1 in longevity provides an interesting link between the circadian clock and ageing.

\section{Clock Mutants and Cancer Association}

Several studies using human subjects lend additional support to the epidemiological data that link defects in circadian rhythms to increased susceptibility for developing cancer and poor prognosis. For example, the expression of all three Per genes is deregulated in breast cancer cells ${ }^{[33]}$. Perl expression is downregulated in most patients, possibly owing to methylation of its promoter. In addition, mutations in NPaS2 have been associated with an increased risk of breast cancer and nonHodgkin's lymphoma ${ }^{[54]}$. Importantly, several studies using mouse models have established convincing links between some clock genes and tumorigenesis. Specifically, Per 1 and Per2 seem to function as tumour suppressors in mice ${ }^{[55,56]}$. Targeted ablation of Per 2 leads to the development of malignant lymphomas ${ }^{[56]}$, whereas its ectopic expression in cancer cell lines results in growth inhibition, cell cycle arrest, apoptosis and loss of clonogenic ability ${ }^{[57]}$. Interestingly, Per 2 mRNA levels are downregulated in several human lymphoma cell lines and in tumour cells from patients with acute myeloid leukaemia ${ }^{[57]}$. overexpression of PER1 can also suppress the growth of human cancer cell lines55. Furthermore, Perl mRNA levels are downregulated in non-small cell lung cancer tissues compared with matched normal tissues ${ }^{[55]}$. In addition, knockdown of CKI $\varepsilon$ induces the growth inhibition of cancer cells, and CKI $\varepsilon$ expression is increased in various human cancers, such as leukaemia and prostate cancer ${ }^{[58]}$. These results consistently point to a direct link between the dysfunction of key circadian regulators and cancer ${ }^{[4]}$.

A matter of debate is whether circadian rhythmicity Per se or only certain core clock genes are involved in cell cycle regulation. So far, only two core clock genes, Perl and Per2, have been shown to function as tumour suppressors in mice. It should be stressed that individual mutation of either Perl or Per2 in the mouse has a mild effect on circadian behaviour ${ }^{[59-62]}$. Fu et al. ${ }^{[56]}$ showed that Per2-mutant mice carrying a deletion in the PAS$\mathrm{b}$ domain have a higher incidence of spontaneous salivary gland hyperplasia than wild-type mice. Moreover, Per2-mutant mice are more sensitive to $\gamma$-irradiation, with significantly higher rates of malignant lymphoma formation after irradiation. Following irradiation, thymocytes from Per2-mutant mice have reduced accumulation of $\mathrm{p} 53$, and thus have reduced apoptosis. Furthermore, the reported $\gamma$-irradiationinduced increase in circadian gene transcription is attenuated in Per2mutant mice ${ }^{[56]}$.

An interesting link between the circadian clock and breast cancer was established in a study demonstrating that PER2 can bind to and destabilize oestrogen receptor- $\alpha(\mathrm{ER} \alpha)^{[63]}$, a key transcription factor that promotes the growth of mammary epithelial cells, deregulation of which has been shown to cause breast cancer ${ }^{[64]}$. Consequently, Per2 overexpression leads to reduced ERá levels and transcriptional activity. Interestingly, all three mammalian Per proteins are structurally similar, with large regions of homology, but with striking differences in specific domains ${ }^{[65]}$. It is possible that specialized protein interactions with these domains could lead to the different regulatory pathways through which PER1 and PER2 exert their tumour suppressor function. This might constitute a system of redundancy so that the two proteins can compensate for each other not only in circadian function but also in tumour suppression activity. As PER1 and PER2 have been shown to heterodimerize $^{[66]}$, it is important to explore whether the concerted contribution of both proteins can elicit increased tumour suppression. Appropriate experiments to address this point by using the Per $1-/-P e r 2-/-$ double-knockout mice have not yet been conducted.

It is important to note that the mutation of one or more core clock genes is itself not necessarily sufficient to elicit increased tumour incidence. In addition, there is no apparent correlation between the disruption of circadian behaviour and increased tumorigenesis in mouse models. Indeed, Cry1-/-Cry2-/- mice67 or lock/Clockmutant mice (which lack exon 19 of Clock) ${ }^{[68]}$, the circadian rhythms of which are highly compromised, o not show a predisposition to cancer on irradiation. Moreover, MEFs derived from Clock/Clockmutant mice have lower levels of DNA synthesis and cell proliferation than wild-type $\mathrm{MEFs}^{[69]}$. Somewhat unexpectedly, abla- 
tion in the mouse of both Cry genes n a Trp53-/- background delays the onset of cancer ${ }^{[70]}$. These observations suggest that other regulatory features intrinsic to clock regulators and independent of their circadian function could participate in carcinogenesis.

Is the disruption of circadian rhythms linked to carcinogenesis?

It seems that individual core circadian clock proteins (such as PER1 and PER2) might have acquired multiple roles and so controls both circadian rhythms and the cell cycle. Also, the consequence of circadian disruption for cancer predisposition might be dependent on how the rhythm is disrupted. Indeed, the molecular mechanism whereby the circadian clock influences cancer development and progression could be explained by its regulation of the cell cycle, DNA damage responses and cellular metabolism.

\section{Cell Cycle Regulation}

The circadian clock shares several conceptual and molecular similarities with the cell cycle ${ }^{[71]}$, both consist of interlocked auto-regulatory loops, and they both rely on sequential phases of transcription, translation, posttranslational odification and degradation. For some cells, the cell cycle itself seems to be synchronized with circadian time as the various phases of the cell cycle follow a circadian programme ${ }^{[72,73]}$. This link is further illustrated by the fact that clock-controlled genes include those that have an essential role in cell cycle control. The circadian regulation of genes encoding key cell cycle regulators, such as Weel (G2/M transition) ${ }^{[5]}$, myC $(\mathrm{G} 0 /$ G1 transition $)^{[56]}$ and cyclin d1 (CCND1; G1/S transition $)^{[56]}$, has been demonstrated in mammals, and light induces the expression of Weel in zebrafish74. Weel is a kinase that phosphorylates and inactivates the cell division cycle 2 (CdC2)-cyclin b1 complex to control the G2/M transition. Wee1 has robust CLoCK- bMAL1dependent circadian oscillations in the mouse liver ${ }^{[5]}$. Furthermore, partial hepatectomy-induced liver regeneration is impaired in Cry1-/Cry2-/- arrhythmic mice, which also show deregulated expression of Wee $I^{[5]}$. These studies demonstrate that Weel is a key molecular link between the circadian cycle and the cell cycle.

CCND1 is another circadian clock-regulated gene that has been implicated in tumorigenesis, especially in breast cancer. Overexpression of CCND1 induces mam- mary tumorigenesis, and high levels of CCNd1 in ER $\alpha$ positive breast cancer correlates with poor prognosis ${ }^{[75]}$. It would be interesting to determine whether the oscillatory expression of $C C N D 1$ is compromised in cancer. Other genes that regulate cell proliferation, such as growth arrest and DNA damage-inducible protein $\alpha$ $(G a D D 45 a)$ and $m D m 2$, also have a circadian pattern of expression, which is abolished in Per2-mutant mice56. Importantly, $m y C$ is a clock-regulated gene, the circadian expression of which is completely abolished in Per2-mutant mice56. Intriguingly, the activators NPAS2 and bMAL1 have been shown to repress $m y C$ expres$\operatorname{sion}^{[56]}$, suggesting an as yet undeciphered pathway of transcriptional control. A microarray analysis of mouse liver and skeletal muscle identified several cell cycle and proliferation regulators, such as cyclin-dependent kinase inhibitor 1A (CDKN1a), which encodes p21, platelet-derived growth factor receptor $(P D G F r)$, vascular endothelial growth factor $(V e G F)$ and KIT ligand $(K I T l G)$, that displayed circadian oscillations in expres$\operatorname{sion}^{[69]}$.

Although these connections between the circadian clock and regulators of the cell cycle have been observed, it is important to note that other evidence shows that the link between the circadian clock and cell proliferation is not absolute. For example, several mouse models with defects in the circadian clock (such as Cry1-/-Cry2-/- mice ${ }^{[67]}$ or Clock/Clockmutant mice ${ }^{[68]}$ ) do not show growth defects during embryogenesis or increased incidences of cancer. Therefore, we propose that the circadian control of cell proliferation is more opportunistic, depending on the physiological state of a cell, and should thereby be considered a fine-tuning mechanism. One example of the above concept is illustrated by the impairment in the partial hepatectomy-induced liver regeneration in Cryl$/$ Cry $2-/-$ mice $^{[5]}$, in which a functional circadian clock might prove advantageous in adverse conditions. Nevertheless, various studies suggest that at least some of the key cell cycle genes are under circadian regulation. It is therefore possible that alterations in circadian rhythms might lead to cancer through the deregulated expression of these cell cycle genes.

\section{DNA Damage Responses}

Damage to DNA, by either intracellular agents (such 
as metabolic by-products) or external agents (such as ionizing radiation), can cause cancer. However, cells have evolved several mechanisms to repair damaged DNA. Recent results suggest that one such repair mechanism, nucleotide excision repair, displays circadian oscillation in mouse brain, possibly through oscillations in the expression of the DNA damage recognition protein for this pathway, Xeroderma pigmentosum A $(\mathrm{XPA})^{[76]}$. XPA levels also oscillate in mouse liver ${ }^{[76]}$, suggesting that circadian nucleotide excision repair might be operating in peripheral tissues.

PER1 also seems to function as a tumour suppressor by regulating cell cycle genes and interacting with key DNA damage-activated checkpoint proteins ${ }^{[55]}$. Perl overexpression in cancer cells increases ionizing radiation-induced apoptosis, whereas its knockdown has protective effects. Ionizing radiation leads to PER1 nuclear translocation and the induction of $m y C$ expression. Interestingly, PER1 interacts with the DNA doublestrand break-activated kinases ataxia telangiectasia mutated (ATM) and CHK2 ${ }^{[55]}$. Therefore, PER1 can function as a tumour suppressor by activating multiple pathways, including the DNA damage response. Another circadian protein, timeless (TIM), which is necessary for the robustness of rhythmicity ${ }^{[77]}$, has been shown to interact with the checkpoint proteins ataxia telangiectasia and Rad3-related (ATR) and $\mathrm{CHK} 1^{[78]}$. Interestingly, this interaction is stimulated by DNA damage, and TIM seems to function as a mediator between sensors and effectors of the DNA damage response. These observations indicate that core clock proteins can interact with checkpoint proteins and could thereby modulate the DNA damage response to increase DNA repair and so protect against tumorigenesis.

Although it is not yet clear whether these interactions are present only at a certain time of the day, the circadian expression of the clock proteins suggests that this is the case. This conclusion has further connotations, as conversely the treatment of cultured cells with DNA damage-inducing agents affects rhythmicity by resetting the $\operatorname{clock}^{[79,80]}$. An additional important twist was revealed by experiments in $N$. crassa, a filamentous ungus, the circadian clock of which has been extensively studied. The $N$. crassa clock gene Period 4 (Prd4), an orthologue of mammalian $\mathrm{CHK}_{2}{ }^{[81]}$, phosphorylates the clock protein frequency (FRQ), an event that has a crucial role in resetting the clock in N. crassa.
Importantly, DNA damage resets the clock in a PRd4dependent manner. Similarly, DNA damage can cause phase advances in the circadian rhythms in a dose- and time-dependent manner in mammals ${ }^{[79,80]}$. These studies, therefore, emphasize the fact that DNA damage can also modulate circadian rhythms, and could in fact function as a zeitgeber. From an evolutionary point of view, this could be a mechanism that has evolved so that cells could avoid DNA replication during times of high ultraviolet light exposure or during periods of intense metabolism when DNA damaging by-products are abundant. Such gating of cell division is remarkably evident in the budding yeast Saccharomyces cerevisiae. This organism has ultradian metabolic cycles that are temporally separated from the cell division cycle ${ }^{[82,83]}$. Cell division takes place exclusively during the reductive phase of the metabolic cycle to prevent DNA damage from oxidative stress. It is conceivable that uncoupling of this delicate balance could induce DNA damage, predisposing cells to tumorigenesis.

\section{Metabolism}

Cancer cells have a wide array of metabolic abnormalities. Accumulating evidence has revealed an intimate relationship between circadian rhythms and metabolism ${ }^{[84]}$. Although the circadian clock regulates multiple metabolic pathways, metabolite availability and feeding behaviour can in turn regulate the circadian clock $^{[18]}$. Several genes (the protein products of which have crucial roles in metabolic processes) exhibit a circadian expression pattern; these include glucose-6phosphatase and PCK2 (gluconeogenesis), pyruvate kinase (glycolysis), glucokinase (glycogen synthesis), glucose transporter2 (glucose transport) and HMG-CoA reductase (cholesterol metabolism). Forced misalignment of behavioural and circadian cycles in human subjects was recently shown to cause a decrease in leptin and an increase in glucose and insulin levels ${ }^{[85]}$. Moreover, metabolic defects are observed in animals lacking core clock genes such as Clock and Bmall or clock-controlled genes such as Nocturnin (a deadenylase that is highly expressed at night and which specifically removes poly(A) tails from mRNAs) ${ }^{[86]}$. Clock/Clockmutant mice become hyperphagic and obese and develop classic signs of metabolic syndrome, such as hyperglycaemia, dyslipidemia and hepatic steatosis ${ }^{[38]}$. 
Further demonstrating a close association between the circadian clock and metabolism, the expression of several nuclear receptors, including peroxisome proliferator- activated receptor (PPAR) family members $(\operatorname{PPAR} \alpha, \operatorname{PPAR} \gamma$ and PPAR $\delta)$ and oestrogen-related receptor (ESRR) family members (ESRR $\alpha, E S R R \beta$ and $\operatorname{ESRR} \gamma)$, is rhythmic in a tissue-specific manner ${ }^{[87]}$. PPAR $\gamma$ co-activator $1 \alpha$ (PPARGC $1 \alpha$ ) has a circadian expression pattern in metabolic tissues, and mice lacking Ppargcla have defects in locomotor activity, body temperature regulation and metabolic rate ${ }^{[88]}$. Another example of direct regulation of cellular metabolism by functional CLoCK is observed in the case of liver glucokinase, an enzyme that regulates glycogen synthesis in the liver, the mRNA levels of which oscillate in normal liver but not in Clock/Clock-mutant liver ${ }^{[89]}$. Although we are still lacking a satisfactory molecular understanding of how clock proteins modulate metabolism, emerging evidence implicates chromatin remodelling as a key control mechanism, suggesting that some clock regulators may be at the crossroad between epigenetics and metabolism ${ }^{[51,90]}$. Perturbations in the expression or activities of these regulators (such as CLoCK and SIRT1) could contribute to cancer by causing higher proliferation and defects in metabolic pathways, for example in the modulation of glycolytic and gluconeogenic enzymes.

\subsection{SIRT1 and Cancer}

SIRT1 has crucial roles in metabolism and survival by deacetylating several proteins and regulating gene expression through histone deacetylation. A fascinating hallmark of SIRT1 is that its enzymatic activity is NAD+-dependent ${ }^{[91]}$. As the NAD+/NADH ratio is a direct measure of the energy status of a cell, the NAD+ dependence of SIRT1 directly links cellular energy metabolism and the deacetylation of target proteins. SIRT1 has been reported to both promote and suppress tumour growth ${ }^{[92]}$, briefly, SIRT1 deacetylates p53 and so inhibits its activity ${ }^{[93]}$, resulting in reduced apoptosis after genotoxic stress. SIRT1 also interacts with hypermethylated in cancer 1 (HIC1) ${ }^{[94]}$, a tumour suppressor that is epigenetically silenced in an age-dependent manner. SIRT1 is upregulated in tumours that lack HIC1, and prevents apoptosis by deacetylating p53. SIRT1 can also promote cancer cell survival by deacetylating the DNA repair protein $\mathrm{Ku} 70$. deacetylation of $\mathrm{Ku} 70$ blocks mitochondrial translocation of BAX and prevents apoptosis, thereby promoting tumour progression ${ }^{[95]}$. Conversely, SIRT1 can also function as a tumour suppressor. Deficiency of SIRT1 in mice impairs the DNA damage response, which leads to genomic instability ${ }^{[96]}$. Moreover, induction of double-strand breaks causes SIRT1 to relocalize from repetitive DNA sequences and gene promoters to the site of DNA damage to facilitate repair ${ }^{[97]}$. Furthermore, ectopic induction of SIRT1 suppresses intestinal tumorigenesis and colon cancer growth in vivo ${ }^{[98]}$, by deacetylating and thereby inactivating $\beta$-catenin, SIRT1 can lead to reduced cell proliferation. It seems evident that SIRT1 can promote or prevent cancer depending on the specific function of its substrate. SIRT1 also deacetylates, and thereby regulates, several metabolic proteins. It deacetylates PPARGC1 $\alpha$ and forkhead box ol (FoXol) to increase gluconeogenesis ${ }^{[99]}$. In addition, SIRT1 interacts with SMRT, thus inhibiting the transcription of PPAR $\gamma$ target genes. It thereby promotes fat mobilization from white adipocytes ${ }^{[100]}$. SIRT1 also deacetylates and activates liver $\mathrm{X}$ receptor (LXR), which leads to regulation of cholesterol metabolism by transactivating the ATP-binding cassette transporter A1 (aBCal) promoter ${ }^{[101]}$. AbCA1 regulates cholesterol efflux from peripheral tissues to form high-density lipoprotein (HdL), which is cleared by the liver. Further studies are needed to ascertain whether the metabolic defects observed in various cancers could be attributed to alterations in SIRT1 function. The NAD+ connection. Genes encoding circadian clock proteins are regulated by epigenetic mechanisms, such as histone phosphorylation, acetylation and methylation, which have been shown to follow a circadian rhythm ${ }^{[48,49,102]}$. All histone posttranslational modifications are directly coupled to cellular metabolism, as these modifications use varying levels of metabolites ${ }^{[103]}$. Circadian oscillation of SIRT1 activity suggested that cellular NAD + levels can also oscillate. Using mass spectrometry-liquid chromatography, our laboratory has demonstrated that NAD+ levels do indeed oscillate in serum-entrained MEFs23. Another study showed that NAD+ levels display bimodal oscillation in mouse liver ${ }^{[24]}$. The circadian clock controls the expression of nicotinamide phosphoribosyltransferase (NAMPT), a key rate-limiting enzyme in the salvage pathway of NAD + biosynthesis $^{[23,24]}$. CLoCK, bMAL1 and SIRT1 are recruited to the NamPT pro- 
moter in a circadian clock-dependent manner. The oscillatory expression of NamPT is abolished in Clock/ Clock-mutant mice, which results in drastically reduced levels of NAD+ in MEFs derived from these mice ${ }^{[23]}$. These results make a compelling case for the existence of an enzymatic and transcriptional feedback loop, in which SIRT1 regulates the levels of its own cofactor. Interestingly, another class of NAD+-dependent enzymes, poly(ADP-ribose) polymerases (PARPs) have been shown to functionally interact with SIRT $1^{[104]}$. PARP1, the best characterized of the family, is activated by DNA damage and has a role in DNA repair ${ }^{[105]}$. Increased activity of PARP1 as a result of DNA damage or PARP1 overexpression depletes the intracellular pool of NAD+, leading to reduced SIRT1 activity and cell death $^{[106]}$. Owing to their role in DNA repair, PARP inhibitors have become attractive drugs against DNA damage response-defective tumours, such as bRCA1- or bRCA2-mutant cancers ${ }^{[107]}$. It will be interesting to test whether the resetting of the circadian clock by DNA damage could be a consequence of PARP1-mediated inhibition of SIRT1. Given the direct control of SIRT1 deacetylase activity by $\mathrm{NAD}+$, circadian regulation of $\mathrm{NAD}+$ levels seems to be a crucial regulatory mechanism controlling circadian rhythms, metabolism and cell growth. Interestingly, altered NAMPT levels have been implicated in metabolic disorders and cancer ${ }^{[108]}$, and FK866 (a highly specific NAMPT inhibitor that abolishes NAD+ circadian oscillations and SIRT1 cyclic activity ${ }^{[23]}$ ) is reported to induce apoptosis in human cancer cells ${ }^{[109]}$. These results suggest that a direct molecular coupling exists between the circadian clock, energy metabolism and cell survival. Further investigation is needed to uncover the precise function of circadian control of SIRT1 activity in the regulation of metabolism and tumorigenesis.

\section{Conclusion}

Circadian control of physiology and behaviour is required for a healthy life. In this Review we have discussed the current literature that supports the idea that cell growth, survival, DNA damage responses and energy metabolism are under circadian control. Circadian clock-mediated transcriptional and post-translational regulations fine-tune the events leading to tissue homeostasis. Core clock proteins have evolved to carry out multiple tasks and so can regulate diverse facets of physiology. The acetyltransferase activity of CLoCK, and the counterbalancing deacetylase activity of SIRT1, seem to regulate and integrate the circadian clock and metabolism. Although direct evidence of defects in a clock-mediated metabolic pathway leading to cancer is still missing, circadian control of NAD + levels might prove to be the missing link. Loss of a functional circadian clock might increase NAD + levels in cancer cells in which NAD+ is used at a higher rate for glycolysis. Moreover, it is also conceivable that CLoCK can influence cellular proliferation and metabolism by acetylating, and so regulating, several non-histone proteins (such as nuclear receptors, cell cycle proteins and checkpoint proteins). SIRT1, which exists in a complex with CLoCK, has both pro-cancer and anticancer effects. This poses a challenge, but also provides opportunities for cancer treatment. Depending $n$ the acetylation status of the SIRT1 substrate in affected cancer tissues, small molecule activators or repressors of SIRT1 could be used as therapeutics. Further studies are needed to identify how the oscillatory nature of SIRT1 activity, NDd + availability and potentially CLoCK activity are modulated in cancer. These could not only further our knowledge of cancer growth and metabolism, but also open new avenues for chronotherapy.

\section{References}

[1] A. Compagni and G. Christofori, "Recent advances in research on multistage tumorigenesis", Br. J. Cancer, Vol. 83, pp. 1-5, 2000.

[2] O. Warburg, "On the origin of cancer cells", Science, Vol. 123, pp. 309-314, 1956.

[3] R. G. Jones and C. B. Thompson, "Tumor suppressors and cell metabolism: a recipe for cancer growth", Genes Dev, Vol. 23, pp. 537-548, 2009.

[4] S. Sahar and P. Sassone-Crosi, "Circadian clock and breast cancer: a molecular link”, Cell Cycle, Vol. 6, pp. 1329-1331, 2007.

[5] T. Matsuo, S. Yamaguchi, S. Mitsui, A. Emi, F. Shimoda, H. Okamura, "Control mechanism of the circadian clock for timing of cell division in vivo", Science, Vol. 302, pp. 255-259, 2003.

[6] C. B. Green, J. S. Takahashi, and J. Bass, "The meter of metabolism", Cell, Vol. 134, pp. 728-742, 2008.

[7] U. Schibler and P. Sassone-Corsi, "A web of 
circadian pacemakers", Cell, Vol. 111, pp. 919-922, 2002.

[8] M. Merrow, K. Spoelstra, and T. Roenneberg, "The circadian cycle: daily rhythms from behaviour to genes", EMBO Rep, Vol. 6, pp. 930-935, 2005.

[9] R. G. Stevens, "Circadian disruption and breast cancer: from melatonin to clock genes", Epidemiology, Vol. 16, pp. 254-258, 2005.

[10] E. S. Schernhammer, F. Laden, F. E. Speizer, W. C. Willett, D. J. Hunter, I. Kawachi, and G, A. Colditz, "Rotating night shifts and risk of breast cancer in women participating in the nurses' health study", J. Natl. Cancer Inst., Vol. 93, pp. 1563-1568, 2001.

[11] J. Hansen, "Increased breast cancer risk among women who work predominantly at night", Epidemiology, Vol. 12, pp. 74-77, 2001.

[12] E. S. Schernhammer, F. Berrino, V. Krogh, G. Secreto, A. Micheli, E. Venturelli, S. Sieri, C. T. Sempos, A. Cavalleri, H. J. Schünemann, S. Strano, and P. Muti, "Urinary 6-sulfatoxymelatonin levels and risk of breast cancer in postmenopausal women”, J. Natl. Cancer Inst., Vol. 100, pp. 898905, 2008.

[13] R. C. Travis, D. S. Allen, I. S. Fentiman, and T. J. Key, "Melatonin and breast cancer: a prospective study", J. Natl. Cancer Inst., Vol. 96, pp. 475-482, 2004.

[14] C. G. Lis, J. F. Grutsch, P. Wood, M. You, I. Rich, and W. J. M. Hrushesky, "Circadian timing in cancer treatment: the biological foundation for an integrative approach", Integr. Cancer Ther., Vol. 2, pp. 105-111, 2003.

[15] F. Levi, C. Focand, A. Karabouéa, V. de la Valettea, D. Focan-Henrardd, B. Baronb, F. Kreutzd, S. Giacchettia, "Implications of circadian clocks for the rhythmic delivery of cancer therapeutics", Adv. Drug Deliv. Rev., Vol. 59, pp. 1015-1035, 2007.

[16] M. Kobayashi, P. A. Wood, and W. J. Hrushesky, "Circadian chemotherapy for gynecological and genitourinary cancers", Chronobiol. Int., Vol. 19, pp. 237-251, 2002.

[17] N. R. Glossop and P. E. Hardin, "Central and peripheral circadian oscillator mechanisms in flies and mammals", J. Cell Sci., Vol. 115, pp. 33693377, 2002.

[18] F. Damiola, N. L. Minh, N. Preitner, B. Kornmann, F. Fleury-Olela, and U. Schibler, "Restricted feeding uncouples circadian oscillators in peripheral tissues from the central pacemaker in the suprachiasmatic nucleus", Genes Dev., Vol. 14, pp. 2950-2961, 2000.
[19] H. Tsubouchi, A. Kamibeppu, K. Fujisaki, J. Nagahama, and S. Hashimoto, "Hepatic gluconeogenic key enzymes in patients with hepatic cancer", Gastroenterol. Jpn., Vol. 15, pp. 564-569, 1980.

[20] M. W. Young and S. A. Kay, "Time zones: a comparative genetics of circadian clocks", Nature Rev. Genet., Vol. 2, pp. 702-715, 2001.

[21] S. Panda, S. Panda, M. P. Antoch, B. H. Miller, A. I. Su, A. B. Schook, M. Straume, P. G. Schultz, S. A. Kay, J. S. Takahashi, J. B. Hogenesch, "Coordinated transcription of key pathways in the mouse by the circadian clock", Cell, Vol. 109, pp. 307-320, 2002.

[22] M. H.Hastings, A. B. Reddy, and E. S. Maywood, "A clockwork web: circadian timing in brain and periphery, in health and disease", Nature Rev. Neurosci., Vol. 4, pp. 649-661, 2003.

[23] Y. Nakahata, S. Sahar, G. Astarita, M. Kaluzova, and P . Sassone-Corsi, "Circadian control of the NAD+ salvage pathway by CLOCK-SIRT1", Science, Vol. 324, pp. 654-657, 2009.

[24] K. M. Ramsey, J. Yoshino, C. S. Brace, D. Abrassart, Y. Kobayashi, B. Marcheva, H.-K. Hong, J. L. Chong, E. D. Buhr, C. Lee, J. S. Takahashi, S Imai, and J. Bass, "Circadian clock feedback cycle through NAMPT-mediated NAD+ biosynthesis", Science, Vol. 324, pp. 651-654, 2009.

[25] L. P. Shearman, M. J. Zylka, D. R. Weaver, L. F. Kolakowski, Jr and S. M. Reppert, "Two period homologs: circadian expression and photic regulation in the suprachiasmatic nuclei”, Neuron, Vol. 19, pp. 1261-1269, 1997.

[26] U. Albrecht, Z. S. Sun, G. Eichele, and C. C. Lee, "A differential response of two putative mammalian circadian regulators, mper1 and mper2, to light", Cell, Vol. 91, pp. 1055-1064, 1997.

[27] A. Balsalobre, F. Damiola, and U. Schibler, "A serum shock induces circadian gene expression in mammalian tissue culture cells", Cell, Vol. 93, pp. 929-937, 1998.

[28] M. Akashi and E. Nishida, "Involvement of the MAP kinase cascade in resetting of the mammalian circadian clock", Genes Dev., Vol. 14, pp. 645-649, 2000.

[29] K. Sanada, T. Okano, and Y. Fukada, "Mitogen-activated protein kinase phosphorylates and negatively regulates basic helix-loop-helix-PAS transcription factor BMAL1”, J. Biol. Chem., Vol. 277, pp. 267271, 2002.

[30] H. S. Shim, H. Kim, J. Lee, G. H. Son, S. Cho, T. H Oh, S. H. Kang, D.-S. Seen, K. H. Lee, and K. 
Kim, "Rapid activation of CLOCK by $\mathrm{Ca}^{2+}$ dependent protein kinase $\mathrm{C}$ mediates resetting of the mammalian circadian clock", EMBO Rep., Vol. 8, pp. 366-371, 2007.

[31] M. Gallego and D. M. Virshup, "Post-translational modifications regulate the ticking of the circadian clock", Nature Rev. Mol. Cell Biol., Vol. 8, pp. 139148, 2007.

[32] K. Kaasik and C. C. Lee, "Reciprocal regulation of haem biosynthesis and the circadian clock in mammals", Nature, Vol. 430, pp. 467-471, 2004.

[33] T. K. Sato, S. Panda1, L. J. Miraglia, T. M. Reyes, R. D. Rudic, P. McNamara, K. A. Naik, G. A. FitzGerald, S. A. Kay, J. B. Hogenesch, "A functional genomics strategy reveals Rora as a component of the mammalian circadian clock", Neuron, Vol. 43, pp. 527-537, 2004.

[34] N. Preitner, F. Damiola1, L. L. Molina, J. Zakany, D. Duboule, U. Albrecht, U. Schibler, "The orphan nuclear receptor REV-ERB $\alpha$ controls circadian transcription within the positive limb of the mammalian circadian oscillator", Cell, Vol. 110, pp. 251-260, 2002.

[35] M. Reick, J. A. Garcia, C. Dudley, and S. L. McKnight, "NPAS2: an analog of clock operative in the mammalian forebrain", Science, Vol. 293, pp. 506509, 2001.

[36] J. P. DeBruyne, D. R. Weaver, and S. M. Reppert, "CLOCK and NPAS2 have overlapping roles in the suprachiasmatic circadian clock", Nature Neurosci., Vol. 10, pp. 543-545, 2007.

[37] J. P. DeBruyne, D. R. Weaver, and S. M. Reppert, "Peripheral circadian oscillators require CLOCK", Curr. Biol., Vol. 17, pp. 538-539, 2007.

[38] F. W. Turek, C. Joshu, A. Kohsaka, E. Lin, G. Ivanova, E. McDearmon, A. Laposky, S. LoseeOlson, A. Easton, D. R. Jensen, R. H. Eckel, J. S. Takahashi, J. Bass, "Obesity and metabolic syndrome in circadian Clock mutant mice", Science, Vol. 308, pp. 1043-1045, 2005.

[39] R. V. Kondratov, A. A. Kondratova, C. Lee, V. Y. Gorbacheva, M. V. Chernov, and M. P. Antoch, "Post-translational regulation of circadian transcriptional CLOCK(NPAS2)/BMAL1 complex by CRYPTOCHROMES", Cell Cycle., Vol. 5, pp. 890-895, 2006.

[40] H. Dardente, E. E. Fortier, V. Martineau, and N. Cermakian, "Cryptochromes impair phosphorylation of transcriptional activators in the clock: a general mechanism for circadian repression", Biochem. J., Vol. 402, pp. 525-536, 2007.
[41] T. Shirogane, J. Jin, X. L. Ang, and J. W. Harper, "SCF $\beta$-TRCP controls clock-dependent transcription via casein kinase 1-dependent degradation of the mammalian period-1 (Per1) protein", J. Biol. Chem., Vol. 280, pp. 26863-26872, 2005.

[42] M. Akashi, Y. Tsuchiya, T. Yoshino, and E. Nishida, "Control of intracellular dynamics of mammalian period proteins by casein kinase I $\varepsilon$ (CKI $\varepsilon$ ) and CKI $\delta$ in cultured cells", Mol. Cell Biol., Vol. 22, pp. 1693-1703, 2002.

[43] L. Busino, F. Bassermann, A. Maiolica, C. Lee, P. M. Nolan, S. I. H. Godinho, G. F. Draetta, M. Pagano, "SCFFbxl3 controls the oscillation of the circadian clock by directing the degradation of cryptochrome proteins", Science, Vol. 316, pp. 900904, 2007.

[44] S. I. H. Godinho, E. S. Maywood, L. Shaw, V. Tucci, A. R. Barnard, L. Busino, M. Pagano, R. Kendall, M. M. Quwailid, M. R. Romero, J. O'Neill, J. E. Chesham, D. Brooker, Z. Lalanne, M. H. Hastings, P. M. Nolan, "The after-hours mutant reveals a role for Fbxl3 in determining mammalian circadian period", Science, Vol. 316, pp. 897-900, 2007.

[45] S. M. Siepka, S.-H. Yoo, J. Park, W. Song, V. Kumar, Y. Hu, C. Lee, J. S. Takahashi, "Circadian mutant Overtime reveals F-box protein FBXL3 regulation of cryptochrome and period gene expression", Cell, Vol. 129, pp. 1011-1023, 2007.

[46] L. Cardone, J Hirayama, F. Giordano, T. Tamaru, J. J. Palvimo, P. Sassone-Corsi, "Circadian clock control by SUMOylation of BMAL1", Science, Vol. 309, pp. 1390-1394, 2005.

[47] J. Hirayama, S. Sahar, B. Grimaldi, T. Tamaru, K. Takamatsu, Y. Nakahata, and P. Sassone-Corsi, "CLOCK-mediated acetylation of BMAL1 controls circadian function", Nature, Vol. 450, pp. 10861090, 2007.

[48] J. P. Etchegaray, C. Lee, P. A. Wade, and S. M. Reppert, "Rhythmic histone acetylation underlies transcription in the mammalian circadian clock", Nature, Vol. 421, pp. 177-182, 2003.

[49] J. A. Ripperger and U. Schibler, "Rhythmic CLOCKBMAL1 binding to multiple E-box motifs drives circadian Dbp transcription and chromatin transitions" Nature Genet., Vol. 38, pp. 369-374, 2006.

[50] M. Doi, J. Hirayama, and P. Sassone-Corsi, "Circadian regulator CLOCK is a histone acetyltransferase", Cell, Vol. 125, pp. 497-508, 2006.

[51] Y. Nakahata, M. Kaluzova, B. Grimaldi, S. Sahar, J. Hirayama, D. Chen, L. P. Guarente, P. Sassone- 
Corsi, "The NAD+-dependent deacetylase SIRT1 modulates CLOCK-mediated chromatin remodeling and circadian control", Cell, Vol. 134, pp. 329-340, 2008.

[52] G. Asher, D. Gatfield, M. Stratmann, H. Reinke, C. Dibner, F. Kreppel, R. Mostoslavsky, F. W. Alt, U. Schibler, "SIRT1 regulates circadian clock gene expression through PER2 deacetylation", Cell, Vol. 134, pp. 317-328, 2008.

[53] S. Y. Chen, S.-T. Chen, K.-B. Choo, M.-F. Hou, K.T. Yeh, S.-J. Kuo, and J.-G. Chang, "Deregulated expression of the PER1, PER2 and PER3 genes in breast cancers", Carcinogenesis, Vol. 26, pp. 1241246, 2005.

[54] A. E. Hoffman, T. Zheng, Y. Ba, and Y. Zhu, "The circadian gene NPAS2, a putative tumor suppressor, is involved in DNA damage response", Mol. Cancer Res., Vol. 6, pp. 1461-1468, 2008.

[55] S. Gery, N. Komatsu, L. Baldjyan, A. Yu, D. Koo, H. P. Koeffler, "The circadian gene perl plays an important role in cell growth and DNA damage control in human cancer cells", Mol. Cell, Vol. 22, pp. 375-382, 2006.

[56] L. Fu, H. Pelicano, J. Liu, P. Huang, and C. Lee, "The circadian gene Period2 plays an important role in tumor suppression and DNA damage response in vivo", Cell, Vol. 111, pp. 41-50, 2002.

[57] S. Gery, A. F. Gombart, W. S. Yi, C. Koeffler, W.K. Hofmann, and H. P. Koeffler, "Transcription profiling of C/EBP targets identifies Per2 as a gene implicated in myeloid leukemia", Blood, Vol.106, pp. 2827-2836, 2005.

[58] W. S. Yang and B. R. Stockwell, "Inhibition of casein kinase 1-epsilon induces cancer-cell-selective, PERIOD2-dependent growth arrest", Genome Biol., Vol. 9, pp. 92, 2008.

[59] N. Cermakian, L. Monaco, M. P. Pando, A. Dierich, and P. Sassone-Corsi, "Altered behavioral rhythms and clock gene expression in mice with a targeted mutation in the Period1 gene", EMBO J., Vol. 20, pp. 3967-3974, 2001.

[60] B. Zheng, U. Albrecht, K. Kaasik, M. Sage, W. Lu, S. Vaishnav, Q. Li, Z. S. Sun, G. Eichele, A. Bradley, C. C. Lee, "Nonredundant roles of the mPer1 and mPer2 genes in the mammalian circadian clock", Cell, Vol. 105, pp. 683-694, 2001.

[61] K. Bae, X. Jin, E. S. Maywood, M. H. Hastings, S. M. Reppert, and D. R. Weaver, "Differential functions of mPer1, mPer2, and mPer3 in the SCN circadian clock", Neuron, Vol.30, pp. 525-536, 2001.

[62] B. Zheng, D. W. Larkin, U. Albrecht, Z. S. Sun, M.
Sage, G. Eichele, C. C. Lee, and A. Bradley, "The mPer2 gene encodes a functional component of the mammalian circadian clock", Nature, Vol. 400, pp. 169-173, 1999.

[63] S. Gery, R. K.Virk, K. Chumakov, A. Yu, and H. P. Koeffler, "The clock gene Per2 links the circadian system to the estrogen receptor", Oncogene, Vol. 26, pp. 7916-7920, 2007.

[64] K. A. Green and J. S. Carroll, "Oestrogen-receptormediated transcription and the influence of co-factors and chromatin state", Nature Rev. Cancer, Vol. 7, pp. 713-722, 2007.

[65] J. Hirayama and P. Sassone-Corsi, "Structural and functional features of transcription factors controlling the circadian clock", Curr. Opin. Genet. Dev., Vol. 15, pp. 548-556, 2005.

[66] M. J. Zylka, L. P. Shearman, J. D. Levine, X. Jin, D. R. Weaver, S. M. Reppert, "Molecular analysis of mammalian timeless", Neuron, Vol. 21, pp. 11151122, 1998.

[67] M. A. Gauger and A. Sancar, "Cryptochrome, circadian cycle, cell cycle checkpoints, and cancer", Cancer Res., Vol. 65, pp. 6828-6834, 2005.

[68] M. P. Antoch, V. Y. Gorbacheva, O. Vykhovanets, I. A. Toshkov, R. V. Kondratov, A. A. Kondratova, C. Lee, and A. Y. Nikitin, "Disruption of the circadian clock due to the Clock mutation has discrete effects on aging and carcinogenesis", Cell Cycle, Vol. 7, pp. 1197-1204, 2008.

[69] B. H. Miller, B. H. Miller, E. L. McDearmon, S. Panda, K. R. Hayes, J. Zhang, J. L. Andrews, M. P. Antoch, J. R. Walker, K. A. Esser, J. B. Hogenesch, and J. S. Takahashi, "Circadian and CLOCK-controlled regulation of the mouse transcriptome and cell proliferation", Proc. Natl. Acad. Sci, Vol. 104, pp. 3342-3347, 2007.

[70] N. Ozturk, J. H. Lee, S. Gaddameedhi, and A. Sancar, "Loss of cryptochrome reduces cancer risk in p53 mutant mice", Proc. Natl. Acad. Sci., Vol. 106, pp. 2841-2846, 2009.

[71] T. Hunt and P. Sassone-Corsi, "Riding tandem: circadian clocks and the cell cycle", Cell, Vol. 129, pp. 461-464, 2007.

[72] L. Canaple, T. Kakizawa, and V. Laudet, "The days and nights of cancer cells", Cancer Res., Vol. 63, pp. 7545-7552, 2003.

[73] L. Rensing and K. Goedeke, "Circadian rhythm and cell cycle: possible entraining mechanisms", Chronobiologia, Vol. 3, pp. 853-865, 1976.

[74] J. Hirayama, L. Cardone, M. Doi, and P. SassoneCorsi, "Common pathways in circadian and cell 
cycle clocks: light-dependent activation of Fos/AP1 in zebrafish controls CRY-1a and WEE-1", Proc. Natl. Acad. Sci., Vol. 102, pp. 10194-10199, 2005.

[75] P. G. Roy and A. M. Thompson, "Cyclin D1 and breast cancer”, Breast, Vol. 15, pp. 718-727, 2006.

[76] T. H. Kang, J. T. Reardon, M. Kemp, and A. Sancar, "Circadian oscillation of nucleotide excision repair in mammalian brain", Proc. Natl. Acad. Sci., Vol. 106, pp. 2864-2867, 2009.

[77] J. W. Barnes, S. A. Tischkau, J. A. Barnes, J. W. Mitchell, P. W. Burgoon, J. R. Hickok, and M. U. Gillette, "Requirement of mammalian Timeless for circadian rhythmicity", Science, Vol. 302, pp. 439442, 2003.

[78] K. Unsal-Kacmaz, T. E. Mullen, W. K. Kaufmann, and A. Sancar, "Coupling of human circadian and cell cycles by the timeless protein", Mol. Cell Biol., Vol. 25, pp. 3109-3116, 2005.

[79] M. Oklejewicz, E. Destici, F. Tamanini, R. A. Hut, R. Janssens, G. T.J. van der Horst, "Phase resetting of the mammalian circadian clock by DNA damage", Curr. Biol., 18, pp. 286-291, 2008.

[80] J. J. Gamsby, J. J. Loros, and J. C. Dunlap, “A phylogenetically conserved DNA damage response resets the circadian clock", J. Biol. Rhythms, Vol. 24, pp. 193-202, 2009.

[81] A. M. Pregueiro, Q. Liu, C. L. Baker, J. C. Dunlap, and J. J. Loros, "The Neurospora checkpoint kinase 2: a regulatory link between the circadian and cell cycles", Science, Vol. 313, pp. 644-649, 2006.

[82] R. R. Klevecz, J. Bolen, G. Forrest, and D. B. Murray, "A genomewide oscillation in transcription gates DNA replication and cell cycle", Proc. Natl. Acad. Sci., Vol. 101, pp. 1200-1205, 2004.

[83] Z. Chen, E. A. Odstrcil, B. P. Tu, and S. L. McKnight, "Restriction of DNA replication to the reductive phase of the metabolic cycle protects genome integrity”, Science, Vol. 316, pp. 1916-1919, 2007.

[84] K. Eckel-Mahan, and P. Sassone-Corsi, "Metabolism control by the circadian clock and vice versa", Nature Struct. Mol. Biol., Vol. 16, pp. 462-467, 2009.

[85] F. A. Scheer, M. F. Hilton, C. S. Mantzoros, and S. A. Shea, "Adverse metabolic and cardiovascular consequences of circadian misalignment", Proc. Natl. Acad. Sci., Vol. 106, pp. 4453-4458, 2009.

[86] C. B. Green, N. Douris, S. Kojima , C. A. Strayer, J. Fogerty, D. Lourim, S. R. Keller, and J. C. Besharse, "Loss of Nocturnin, a circadian deadenylase, confers resistance to hepatic steatosis and diet-induced obesity", Proc. Natl. Acad. Sci., Vol. 104, pp. 9888-9893, 2007.
[87] X. Yang, M. Downes, R. T. Yu, A. L. Bookout, W. He, M. Straume, D. J. Mangelsdorf, R. M. Evans, "Nuclear receptor expression links the circadian clock to metabolism", Cell, Vol. 126, pp. 801-810, 2006.

[88] C. Liu, S. Li, T. Liu, J. Borjigin, and J. D. Lin, "Transcriptional coactivator PGC- $1 \alpha$ integrates the mammalian clock and energy metabolism", Nature, Vol. 447, pp. 477-481, 2007.

[89] K. Oishi, K. Miyazakia, K. Kadota, R. Kikuno, T. Nagase, G.Atsumi, N. Ohkura, T. Azama, M. Mesaki, S. Yukimasa, H. Kobayashi, C. Iitaka, T. Umehara, M. Horikoshi, T. Kudo, Y. Shimizu, M. Yano, M. Monden, K. Machida, J. Matsuda, S. Horie, T. Todo, and N. Ishida, "Genome-wide expression analysis of mouse liver reveals CLOCKregulated circadian output genes", J. Biol. Chem., Vol. 278, pp. 41519-41527, 2003.

[90] T. Alenghat, K. Meyers, S. E. Mullican, K. Leitner, A. Adeniji-Adele, J. Avila, M. Buan, R. S. Ahima, K. H. Kaestner, and M. A. Lazar, "Nuclear receptor corepressor and histone deacetylase 3 govern circadian metabolic physiology", Nature, 456, pp. 997-1000, 2008.

[91] S. Imai, C. M. Armstrong, M. Kaeberlein, and L. Guarente, "Transcriptional silencing and longevity protein Sir2 is an NAD-dependent histone deacetylase", Nature, Vol. 403, pp. 795-800, 2000.

[92] C. L. Brooks and W. Gu, "How does SIRT1 affect metabolism, senescence and cancer?", Nature Rev. Cancer, Vol. 9, pp. 123-128, 2009.

[93] H. Vaziri, S. K. Dessain, E. N. Eaton, S.-I. Imai, R. A. Frye, T. K. Pandita, L. Guarente, R. A. Weinberg, "hSIR2SIRT1 functions as an NADdependent p53 deacetylase", Cell, Vol. 107, pp. 149-159, 2001.

[94] W. Y. Chen, D. H. Wang, R. W. C. Yen, J. Luo, W. $\mathrm{Gu}, \mathrm{S}$. B. Baylin, "Tumor suppressor HIC1 directly regulates SIRT1 to modulate p53-dependent DNAdamage responses", Cell, 123, pp. 437-448, 2005.

[95] H. Y. Cohen, S. Lavu, K. J. Bitterman, B. Hekking, T. A. Imahiyerobo, C. Miller, R. Frye, H. Ploegh, B. M. Kessler, D. A. Sinclair, "Acetylation of the $\mathrm{C}$ terminus of Ku70 by CBP and PCAF controls Bax-mediated apoptosis", Mol. Cell, Vol. 13, pp. 627-638, 2004.

[96] R. H. Wang, K. Sengupta, C. Li, H.-S. Kim, L. Cao, C. Xiao, S. Kim, X. Xu, Y. Zheng, B. Chilton, R. Jia, Z.-M. Zheng, E. Appella, X. W. Wang, T. Ried, and C.-X. Deng, "Impaired DNA damage response, genome instability, and tumorigenesis in SIRT1 mutant mice”, Cancer Cell, 14, pp. 312-323, 2008. 
[97] P. Oberdoerffer, S. Michan, M. McVay, R. Mostoslavsky, J. Vann, S.-K. Park, A. Hartlerode, J. Stegmuller, A. Hafner, P. Loerch, S. M. Wright, K. D. Mills, A. Bonni, B. A. Yankner, R. Scully, T. A. Prolla, F. W. Alt, and D. A. Sinclair, "SIRT1 redistribution on chromatin promotes genomic stability but alters gene expression during aging", Cell, Vol. 135, pp. 907-918, 2008.

[98] R. Firestein, G. Blander, S. Michan, P. Oberdoerffer, S. Ogino, J. Campbell, A. Bhimavarapu, S. Luikenhuis, R. de Cabo, C. Fuchs, W. C. Hahn, L. P. Guarente, and D. A. Sinclair, "The SIRT1 deacetylase suppresses intestinal tumorigenesis and colon cancer growth", PLoS ONE Vol. 3, pp. e2020, 2008.

[99] B. Schwer and E. Verdin, "Conserved metabolic regulatory functions of sirtuins", Cell Metab., Vol. 7, pp. 105-112, 2008.

[100] F. Picard, M. Kurtev, N. Chung, A. Topark-Ngarm, T. Senawong, R. M. de Oliveira, M. Leid, M. W. McBurney, and L. Guarente, "Sirt1 promotes fat mobilization in white adipocytes by repressing PPAR- $\gamma "$, Nature, Vol. 429, pp. 771-776, 2004.

[101] X. Li, S. Zhang, G. Blander, J. G. Tse, M. Krieger, and L. Guarente, "SIRT1 deacetylates and positively regulates the nuclear receptor LXR", Mol. Cell, Vol. 28, pp. 91-106, 2007.

[102] C. Crosio, N. Cermakian, C. D. Allis, and P. Sassone-Corsi, "Light induces chromatin modification in cells of the mammalian circadian clock", Nature Neurosci., Vol. 3, pp. 1241-1247, 2000.

[103] E. Borrelli, E. J. Nestler, C. D. Allis, and P. Sassone-Corsi, "Decoding the epigenetic language of neuronal plasticity", Neuron Vol. 60, pp. 961-974, 2008.

[104] U. Kolthur-Seetharam, F. Dantzer, M. W. McBurney, G. de Murcia, G. and P. Sassone-Corsi, "Control of AIF- mediated cell death by the functional interplay of SIRT1 and PARP-1 in response to
DNA damage", Cell Cycle, Vol. 5, pp. 873-877, 2006.

[105] F. Dantzer, J. C. Amé, V. Schreiber, J. Nakamura, M. J. Ménissier-de, and G. de Murcia, "Poly(ADPribose) polymerase-1 activation during DNA damage and repair", Methods Enzymol., Vol. 409, pp. 493-510, 2006.

[106] J. B.Pillai, A. Isbatan, S. Imai, and M. P. Gupta, "Poly(ADP-ribose) polymerase-1-dependent cardiac myocyte cell death during heart failure is mediated by NAD + depletion and reduced $\operatorname{Sir} 2 \alpha$ deacetylase activity", J. Biol. Chem., Vol. 280, pp. 43121-43130, 2005.

[107] S. A. Martin, C. J. Lord, and A. Ashworth, "DNA repair deficiency as a therapeutic target in cancer", Curr. Opin. Genet. Dev., Vol. 18, pp. 80-86, 2008.

[108] A. Garten, S. Petzold, A. Korner, S. I. Imai, and W. Kiess, "Nampt: linking NAD biology, metabolism and cancer", Trends Endocrinol. Metab., Vol. 20, pp. 130-138, 2008.

[109] M. Hasmann and T. Schemainda, "FK866, a highly specific noncompetitive inhibitor of nicotinamide phosphoribosyl transferase, represents a novel mechanism for induction of tumor cell apoptosis", Cancer Res., Vol. 63, pp. 7436-7442, 2003.

[110] M. F.Demierre, P. D. Higgins, S. B. Gruber, E. Hawk, and S. M. Lippman, "Statins and cancer prevention", Nature Rev. Cancer, Vol. 5, pp. 930942, 2005.

[111] M. S. Brown, J. L. Goldstein, and J. M. Dietschy, "Active and inactive forms of 3-hydroxy-3-methylglutaryl coenzyme A reductase in the liver of the rat. Comparison with the rate of cholesterol synthesis in different physiological states", J. Biol. Chem., Vol. 254, pp. 5144-5149, 1979.

[112] R, R. Klevecz, R. M. Shymko, D. Blumenfeld, and P. S. Braly, "Circadian gating of S phase in human ovarian cancer", Cancer Res., Vol. 47, pp. 62676271, 1987. 